\title{
On Terai's exponential equation with two finite integer parameters
}

\author{
Takafumi Miyazaki \\ Division of Pure and Applied Science \\ Faculty of Science and Technology, Gunma University \\ 1-5-1 Tenjin-cho, Kiryu, Gunma, Japan \\ e-mail: tmiyazaki@gunma-u.ac.jp
}

Received: 6 February 2018

Accepted: 25 December 2018

Abstract: Let $r$ be an integer with $r>1$, and $m$ be an even positive integer. Define integers
$A$ and $B$ by the equation $A+B \sqrt{-1}=(m+\sqrt{-1})^{r}$. It is proven by F. Luca in 2012 that the
equation $|A|^{x}+|B|^{y}=\left(m^{2}+1\right)^{z}$ does not hold for any triple $(x, y, z)$ of positive integers not
equal to $(2,2, r)$, whenever $r$ or $m$ exceeds some effectively computable absolute constant. In our
previous work, we estimated this constant explicitly. Here that estimate is substantially improved. Keywords: Exponential Diophantine equation, Linear forms in logarithms of algebraic numbers. 2010 Mathematics Subject Classification: 11D61, 11J86.

\section{Introduction}

Consider the Diophantine equation of the following form:

$$
a^{x}+b^{y}=c^{z},
$$

where base numbers $a, b, c$ are fixed positive integers, and exponents $x, y, z$ are positive integer variables. A natural setting for the parameters of the above equation is that $a, b, c$ are pairwise coprime and greater than the unity. Indeed, the problem is always considered under this condition in the sequel.

It is regarded that equation (1) is of the form that a sum of two integers whose prime factors belong to a fixed set is equal to an integer having the same property on that set. Thus, equation 
(1) is a special example of unit equations, and then the number and magnitude of its solutions can be deduced by the theory of Diophantine approximation. Especially, the theory of linear forms in logarithms, so called Baker's method, is applied to give us an upper estimate of the size of solutions which is effectively computable and depends only on the base numbers.

There are a number of studies on equation (1) in literature. Most of the existing results on equation (1) concern the problem of determining all its solutions for various families of triples $(a, b, c)$. In general, it is not easy to solve the equation even for very particular triples, even though one can find an upper bound for the solutions as mentioned before.

The theme of this note is to study a problem proposed by Terai [18], which origins at his paper published in 1994 (cf. [15]). Here, let us state his conjecture (actually, with some modification (cf. $[2,8]))$ as follows.

Conjecture 1.1. Let $a, b, c$ be pairwise coprime integers greater than 1. Assume that there exists some solution $(x, y, z)$ of Diophantine equation (1) with $\min \{x, y, z\}>1$. Then, it is the only positive solution of Diophantine equation (1), except for the following cases:

$$
\begin{aligned}
& \{a, b\}=\{2,7\}, c=3 ; \\
& \{a, b\}=\left\{2,2^{p-2}-1\right\}, c=2^{p-2}+1,
\end{aligned}
$$

where $p$ is any integer satisfying $p \geqq 4$.

The exceptional cases come from the following equalities:

$$
\begin{aligned}
& 2^{5}+7^{2}=3^{4} ; 2+7=3^{2}, \\
& 2^{p}+\left(2^{p-2}-1\right)^{2}=\left(2^{p-2}+1\right)^{2} ; 2+\left(2^{p-2}-1\right)=\left(2^{p-2}+1\right) .
\end{aligned}
$$

There are so many works concerning Conjecture 1.1, however, it is still unsolved in general. The required assumption on $a, b, c$ in the conjecture can be rephrased as that the equation $a^{p}+b^{q}=c^{r}$ holds for some integers $p, q, r$ with $\min \{p, q, r\}>1$. It is natural to think that investigating exponents $(p, q, r)$ for which there exist infinitely many triples $(a, b, c)$ corresponding to $(p, q, r)$ is essential. The easiest one among such exponents is that $p=q=r=2$, that is, $(a, b, c)$ forms a primitive Pythagorean triple, where Conjecture 1.1 is nothing but the conjecture of Jeśmanowicz [5]. In a series of his papers [15, 16, 17], as an analogy to Jeśmanowicz' conjecture, Terai started to study equation (1) when $a, b, c$ satisfy the equation $a^{2}+b^{2}=c^{r}$ with some odd $r$, in particular, $r \in\{3,5\}$. After this pioneer work, many authors have actively studied the case where $p=q=2$. Actually, the present paper also concerns this case.

Here and hereinafter, let us always consider the case where $p=q=2$ and $r \geqq 2$. In this case, it is helpful to use the following parameterization of $a, b, c$ satisfying $a^{2}+b^{2}=c^{r}$ :

$$
a=\left|\operatorname{Re}(m+n \sqrt{-1})^{r}\right|, \quad b=\left|\operatorname{Im}(m+n \sqrt{-1})^{r}\right|, \quad c=m^{2}+n^{2},
$$

where $m, n$ are coprime integers of different parities with $m>n>0$. Most of the existing results concern two special cases, that is, $n=1$ and $m n \equiv 2(\bmod 4)$. For example, see $[3,10,11,12,15,16]$ for the first, $[4,18]$ for the second, and the references therein. The first case will be dealt with in this paper. 
In what follows, assume that $n=1$. Then, Conjecture 1.1 in this special case can be stated as follows.

Conjecture 1.2. For any fixed pair $(r, m)$ of positive integers with $r>1$ and $m$ even, define a, $b, c$ as follows:

$$
a=\left|\operatorname{Re}(m+\sqrt{-1})^{r}\right|, \quad b=\left|\operatorname{Im}(m+\sqrt{-1})^{r}\right|, \quad c=m^{2}+1 .
$$

Then, the Diophantine equation (1) has no positive solution $(x, y, z)$ other than $(x, y, z)=$ $(2,2, r)$.

Let us recall some existing results in the literature concerning this conjecture. Firstly, Lu [10] solved the problem for $r=2$. After many contributions to the case where $r \in\{3,5\}$ by several authors, Cao-Dong [3] solved this case completely. In [12], the author considered the problem when $r$ is even, and he showed that Conjecture 1.2 is true if either $r \equiv 4(\bmod 8)$, or $r \equiv 6$ (mod 8) with some restriction on $r$ and $m$. The following remarkable result was established by Luca [11] in 2012 and it is the most important contribution to the study on Conjecture 1.2, stated as follows.

Proposition 1.1 (Theorem 1, [11]). Conjecture 1.2 is true if $r$ or $m$ exceeds some effectively computable absolute constant.

As mentioned before, it holds that the solutions $x, y, z$ of equation (1) considered in Conjecture 1.2 are bounded by an effective computable constant depending only on the base numbers, that is, $r$ and $m$. Thus, it may be said that Luca almost completely solved Conjecture 1.2.

The tools in the proof of Proposition 1.1 are the theory of linear forms in logarithms and congruence reductions. The key idea is to examine the prime factors of $c=m^{2}+1$. Luca combines some special congruences with the theory of linear forms in two logarithms in both complex and $p$-adic cases to derive several non-trivial information on those factors (under assuming the existence of non-trivial solution), in particular, about their sizes, the number of them and the multiplicative order of 2 modulo them. Although almost parts in his method are largely based on the premise that $n=1$, the author [13] used a simple argument instead of treating the multiplicative order of 2 modulo the prime factors under consideration, to estimate an effective constant stated in Proposition 1.1 explicitly as follows.

Proposition 1.2 (Theorem 1.1, [13]). Assume that

$$
r>10^{74} \text { or } m>10^{34}
$$

Then, Conjecture 1.2 is true.

The main result in this paper is to improve this proposition as follows.

Theorem 1.1. Assume that

$$
r>10^{14} \text { or } m>10^{23} \text {. }
$$

Then, Conjecture 1.2 is true. 
This theorem is deduced from the following result.

Theorem 1.2. Conjecture 1.2 is true in each of the following cases:

(I) $r>10^{10}$ and $m \geqq r$.

(II) $r>10^{14}$ and $r>m$.

The key idea in the proof of Theorem 1.1 is to divide the problem into the cases where $r / m$ is slightly less than $\pi / 2$ and otherwise, which corresponds to (I) and (II) in Theorem 1.2, respectively. In the first case, one can estimate the values of the logarithms of $a$ and $b$ very precisely. Since those values are used in several places to derive an effective estimate stated in Proposition 1.1, this has a great contribution for the purpose. In the second one, one can estimate the prime factors of $c$ from below better than those obtained in the general case, which also has a good contribution. The proof is completed by using these together with the conclusion of Proposition 1.2, improving some rough arguments in [13] and using the theory of linear forms in logarithms in better way.

The organization of this paper is as follows. In the next section, we clarify our situation for considering Conjecture 1.2. Using simple congruences, in Section 3 we find an elementary lower bound for the solutions. In Section 4 , we obtain very exact estimates of $\log a$ and $\log b$ in the case corresponding to (I) in Theorem 1.2. Several results on the theory of linear forms in two logarithms are quoted in Section 5. Using a few of these, in Section 6 we give naive estimates for $\log a$ and $\log b$ in the case corresponding to (II). A bound for some linear forms in two logarithms related to solutions is obtained in Section 7, and it is used to deduce reasonable upper bounds for the solutions in Section 8. Sections 9, 10 and 11 are devoted to the proofs of Theorem 1.2 (I), (II) and Theorem 1.1, respectively.

\section{Preparation}

Let $r$ and $m$ be positive integers satisfying

$$
r>1, \quad m \equiv 0 \quad \bmod 2 \text {. }
$$

Define integers $A=A(r, m)$ and $B=B(r, m)$ as

$$
A=\operatorname{Re}\left[(m+i)^{r}\right], \quad B=\operatorname{Im}\left[(m+i)^{r}\right]
$$

with $i=\sqrt{-1}$. Put

$$
a=|A|, \quad b=|B|, \quad c=m^{2}+1 .
$$

Then $a, b, c$ are pairwise coprime integers $>1$ satisfying

$$
a^{2}+b^{2}=c^{r} .
$$

Under this situation, let us consider the equation:

$$
a^{x}+b^{y}=c^{z}
$$


where $x, y, z$ are positive integer variables.

For any solution $(x, y, z)$ of equation (2), let us write

$$
\Delta=r X-2 z
$$

where

$$
X=\max \{x, y\} .
$$

From [13, Lemms 3.1] it holds that $\Delta \geqq 0$, and the following implication holds.

$$
\Delta=0 \quad \Rightarrow \quad(x, y, z)=(2,2, r) .
$$

Thus, it suffices to find a contradiction when $\Delta>0$.

In what follows, $(x, y, z)$ denotes any solution of equation (2) with $\Delta>0$.

\section{Elementary lower bounds for solutions}

Lemma 3.1. The following inequalities hold.

$$
\begin{array}{ll}
\Delta<\frac{\log \left(c^{r} / a^{2}\right)}{\log c} x, & \text { if } X=x, \\
\Delta<\frac{\log \left(c^{r} / b^{2}\right)}{\log c} y, & \text { if } X=y .
\end{array}
$$

Proof. Since $c^{z}>a^{x}$ by equation (2), if $X=x$ then

$$
\Delta=r x-2 z<r x-2 \cdot \frac{\log a}{\log c} x=\frac{\log \left(c^{r} / a^{2}\right)}{\log c} x .
$$

Similarly, the case where $X=y$ is dealt with by using the trivial inequality $c^{z}>b^{y}$.

Lemma 3.2. The following inequalities hold.

$$
\begin{array}{ll}
\Delta>\min \{y, 2\} \cdot \frac{\log b}{\log c}, & \text { if } X=x, \\
\Delta>\min \{x, 2\} \cdot \frac{\log a}{\log c}, & \text { if } X=y .
\end{array}
$$

Proof. Reducing equation (2) modulo $b^{\min \{y, 2\}}$ yields that

$$
a^{x} \equiv c^{z} \bmod b^{\min \{y, 2\}} .
$$

Since $a^{2}+b^{2}=c^{r}$, one squares the above congruence to find that

$$
c^{r x} \equiv c^{2 z} \bmod b^{\min \{y, 2\}} .
$$

As $c$ is prime to $b$, it follows that

$$
c^{|r x-2 z|} \equiv 1 \quad \bmod b^{\min \{y, 2\}} .
$$

If $X=x$, then, since $\Delta>0$, the left-hand side above is greater than 1 , which yields $c^{|r x-2 z|}-1 \geqq$ $b^{\min \{y, 2\}}$, thereby the stated inequality holds. Similarly, the case where $X=y$ is dealt with by reducing equation (2) modulo $a^{\min \{x, 2\}}$. 
Lemma 3.3. The following inequality holds.

$$
X>\min \{x, y, 2\} \cdot \frac{r \log c-\log 2}{4 \max \left\{W_{a}, W_{b}\right\}},
$$

where

$$
\begin{aligned}
& W_{a}=\frac{r}{2} \log c-\log a(>0), \\
& W_{b}=\frac{r}{2} \log c-\log b(>0) .
\end{aligned}
$$

Proof. Lemmas 3.1 and 3.2 together yield that

$$
\begin{array}{ll}
\frac{x}{\min \{y, 2\}}>\frac{\log b}{\log \left(c^{r} / a^{2}\right)}, & \text { if } X=x \\
\frac{y}{\min \{x, 2\}}>\frac{\log a}{\log \left(c^{r} / b^{2}\right)}, & \text { if } X=y
\end{array}
$$

If $X=x$, then

$$
\frac{x}{\min \{y, 2\}}>\frac{(r / 2) \log c-W_{b}}{r \log c-2 \log a}=\frac{r \log c-2 W_{b}}{4 W_{a}} .
$$

Similarly, if $X=y$ then

$$
\frac{y}{\min \{x, 2\}}>\frac{r \log c-2 W_{a}}{4 W_{b}}
$$

These inequalities together show that

$$
\frac{X}{\min \{x, y, 2\}}>\frac{r \log c-2 \min \left\{W_{a}, W_{b}\right\}}{4 \max \left\{W_{a}, W_{b}\right\}} .
$$

The inequality $\min \left\{W_{a}, W_{b}\right\}<\log \sqrt{2}$ holds as $2 \max \{a, b\}^{2}>c^{r}$.

\section{Estimates of $a$ and $b$ from calculus}

Put

$$
\alpha=m+i
$$

Then $\alpha$ is written in polar form, as follows:

$$
\alpha=c^{1 / 2}(\cos \theta+i \sin \theta),
$$

where

$$
\theta=\operatorname{Arctan}(1 / m) \text {. }
$$

Note that

$$
0.9 / m<\theta<1 / m \text {. }
$$

Also, by the definitions of $a$ and $b$,

$$
\begin{aligned}
a & =\left|\operatorname{Re} \alpha^{r}\right|=c^{r / 2}|\cos r \theta|, \\
b & =\left|\operatorname{Im} \alpha^{r}\right|=c^{r / 2}|\sin r \theta| .
\end{aligned}
$$


Lemma 4.1. Assume that

$$
m>(2 / \pi) r .
$$

Then

$$
a=c^{r / 2} \cos r \theta, \quad b=c^{r / 2} \sin r \theta .
$$

Proof. Since $0<\theta<1 / m$, it follows that

$$
0<r \theta<r / m<\pi / 2 .
$$

This shows the lemma.

Lemma 4.2. Assume that

$$
m \geqq r
$$

Then

$$
\max \left\{W_{a}, W_{b}\right\}<\log (2 m / r)
$$

In particular,

$$
X>\min \{x, y, 2\} \cdot \frac{r \log c-\log 2}{4 \log (2 m / r)} .
$$

Proof. By Lemma 4.1, $W_{a}$ and $W_{b}$ are expressed as follow:

$$
W_{a}=-\log \cos r \theta, \quad W_{b}=-\log \sin r \theta .
$$

Since $0<r \theta<r / m \leqq 1$ and $\theta>0.9 / m$, it follows that

$$
\begin{aligned}
& \cos r \theta>\cos 1>1 / 2, \\
& \sin r \theta>\sin (1) \cdot r \theta>(1 / 2) r / m .
\end{aligned}
$$

All these together yield the first stated inequality. The second one follows from Lemma 3.3.

\section{Linear form in two logarithms}

Here let us list several estimates for linear forms in logarithms in both complex and $p$-adic cases.

For any algebraic number $\alpha$ of degree $d$ over $\mathbb{Q}$, define as usual the absolute logarithmic height of $\alpha$ by the formula

$$
\mathrm{h}(\alpha)=\frac{1}{d}\left(\log c_{0}+\sum_{1 \leqq i \leqq d} \log \max \left\{1,\left|\alpha^{(i)}\right|\right\}\right),
$$

where $c_{0}>0$ is the leading coefficient of the minimal polynomial of $\alpha$ over $\mathbb{Z}$, and $\alpha^{(1)}, \ldots, \alpha^{(d)}$ are the conjugates of $\alpha$ in the filed of complex numbers.

First, some results from Laurent [6] on lower bounds for linear forms in two complex logarithms are presented as follows. Assume that $\alpha_{1}$ and $\alpha_{2}$ are algebraic numbers with $\left|\alpha_{1}\right| \geqq 1$ and $\left|\alpha_{2}\right| \geqq 1$, and let $\log \alpha_{1}$ and $\log \alpha_{2}$ be any determinations of their logarithms. Put

$$
\Lambda=b_{2} \log \alpha_{2}-b_{1} \log \alpha_{1}
$$

where $b_{1}, b_{2}$ are positive integers. Put

$$
D=\left[\mathbb{Q}\left(\alpha_{1}, \alpha_{2}\right): \mathbb{Q}\right] /\left[\mathbb{R}\left(\alpha_{1}, \alpha_{2}\right): \mathbb{R}\right] .
$$


Proposition 5.1 (Theorem 1, [6]). Let $\varrho$ be a real number with $\varrho>1$. Let $a_{1}, a_{2}$ be positive numbers such that

$$
a_{i} \geqq \varrho\left|\log \alpha_{i}\right|-\log \left|\alpha_{i}\right|+2 D \mathrm{~h}\left(\alpha_{i}\right) \quad(i=1,2) .
$$

Let $K, L, R_{1}, R_{2}, S_{1}, S_{2}$ be positive integers with $K \geqq 2$, and $\mu$ be a real number with $1 / 3 \leqq \mu \leqq$ 1. Suppose that

$$
\left\{\begin{array}{l}
\operatorname{Card}\left\{\alpha_{1}^{r} \alpha_{2}^{s} \mid 0 \leqq r<R_{1}, 0 \leqq s<S_{1}\right\} \geqq L \\
\operatorname{Card}\left\{r b_{2}+s b_{1} \mid 0 \leqq r<R_{2}, 0 \leqq s<S_{2}\right\}>(K-1) L
\end{array}\right.
$$

and

$$
K(\sigma L-1) \log \varrho-(D+1) \log N-D(K-1) \log \mathcal{B}-g L\left(R a_{1}+S a_{2}\right)>\varepsilon(N),
$$

where

$$
\begin{gathered}
R=R_{1}+R_{2}-1, \quad S=S_{1}+S_{2}-1, \quad N=K L, \quad g=\frac{1}{4}-\frac{N}{12 R S} \\
\sigma=\frac{1+2 \mu-\mu^{2}}{2}, \quad \mathcal{B}=\frac{(R-1) b_{2}+(S-1) b_{1}}{2}\left(\prod_{k=1}^{K-1} k !\right)^{-2 /\left(K^{2}-K\right)} \\
\varepsilon(N)=2 N^{-1} \log \left(N ! N^{-N+1}\left(e^{N}+(e-1)^{N}\right)\right) .
\end{gathered}
$$

Then

$$
\left|\Lambda^{\prime}\right|>\varrho^{-\mu K L} \quad \text { with } \quad \Lambda^{\prime}=\Lambda \max \left\{\frac{L S e^{L S|\Lambda| /\left(2 b_{2}\right)}}{2 b_{2}}, \frac{L R e^{L R|\Lambda| /\left(2 b_{1}\right)}}{2 b_{1}}\right\} .
$$

Proposition 5.2 (Theorem 2, [6]). Assume that $\alpha_{1}$ and $\alpha_{2}$ are multiplicatively independent. Let $\varrho$ and $\mu$ be real numbers with $\varrho>1$ and $1 / 3 \leqq \mu \leqq 1$. Set

$$
\sigma=\frac{1+2 \mu-\mu^{2}}{2}, \quad \lambda=\sigma \log \varrho .
$$

Let $a_{1}, a_{2}$ be real numbers such that

$$
\begin{aligned}
& a_{i} \geqq \max \left\{1, \varrho\left|\log \alpha_{i}\right|-\log \left|\alpha_{i}\right|+2 D \mathrm{~h}\left(\alpha_{i}\right)\right\} \quad(i=1,2), \\
& a_{1} a_{2} \geqq \lambda^{2} .
\end{aligned}
$$

Let $h$ be a real number such that

$$
h \geqq \max \left\{D\left(\log \left(\frac{b_{1}}{a_{2}}+\frac{b_{2}}{a_{1}}\right)+\log \lambda+1.75\right)+0.06, \lambda, \frac{D \log 2}{2}\right\}+\log \varrho .
$$

Put

Then

$$
H=\frac{h}{\lambda}, \omega=2+2 \sqrt{1+\frac{1}{4 H^{2}}}, \quad \theta=\sqrt{1+\frac{1}{4 H^{2}}}+\frac{1}{2 H} .
$$

$$
\log |\Lambda| \geqq-C a_{1} a_{2} h^{2}-\sqrt{\omega \theta} h-\log \left(C^{\prime} a_{1} a_{2} h^{2}\right)
$$

with

$$
C=C_{0} \mu /\left(\lambda^{3} \sigma\right), \quad C^{\prime}=\sqrt{C_{0} \omega \theta / \lambda^{6}},
$$

where

$$
C_{0}=\left(\frac{\omega}{6}+\frac{1}{2} \sqrt{\frac{\omega^{2}}{9}+\frac{8 \lambda \omega^{5 / 4} \theta^{1 / 4}}{3 \sqrt{a_{1} a_{2} H}}+\frac{4}{3}\left(\frac{1}{a_{1}}+\frac{1}{a_{2}}\right) \frac{\lambda \omega}{H}}\right)^{2}
$$


Proposition 5.3 (Corollary $2 ; m=10,[6])$. Assume that $\alpha_{1}, \alpha_{2}$ are multiplicatively independent positive rational numbers. Let $H_{1}, H_{2}$ be real numbers such that

$$
H_{i} \geqq \max \left\{\mathrm{h}\left(\alpha_{i}\right), \log \alpha_{i}, 1\right\} \quad(i=1,2) .
$$

Put

$$
b^{\prime}=\frac{b_{1}}{H_{2}}+\frac{b_{2}}{H_{1}} .
$$

Then

$$
\log |\Lambda| \geqq-25.2 H_{1} H_{2}\left(\max \left\{\log b^{\prime}+0.38,10\right\}\right)^{2}
$$

Next, let us present a direct consequence of Bugeaud [1, Theorem 2] on lower bounds for linear forms in two $p$-adic logarithms.

For a prime number $p$ and a non-zero rational number $\alpha$, let us denote by $\nu_{p}(\alpha)$ the exponent of $p$ in the prime factorization of $\alpha$.

Assume that $\alpha_{1}$ and $\alpha_{2}$ are multiplicatively independent rational numbers, and put

$$
\Gamma=\alpha_{1}^{b_{1}} \alpha_{2}^{b_{2}}-1
$$

where $b_{1}, b_{2}$ are positive integers. Let $p$ be a prime such that $\nu_{p}\left(\alpha_{1}\right)=0$ and $\nu_{p}\left(\alpha_{2}\right)=0$. Let $g$ be the minimal positive integer such that $\nu_{p}\left(\alpha_{1}^{g}-1\right)>0$ and $\nu_{p}\left(\alpha_{2}^{g}-1\right)>0$.

Proposition 5.4. Assume that there exists an integer E such that

$$
\frac{1}{p-1}<E \leqq \nu_{p}\left(\alpha_{1}^{g}-1\right)
$$

Let $H_{1}, H_{2}$ be real numbers such that

$$
H_{i} \geqq \max \left\{\log \mathrm{h}\left(\alpha_{i}\right), E \log p\right\} \quad(i=1,2) .
$$

Put

$$
b^{\prime}=\frac{b_{1}}{H_{2}}+\frac{b_{2}}{H_{1}} .
$$

If either $p \geqq 5$, or $p=2$ with $\nu_{2}\left(\alpha_{2}-1\right) \geqq 2$, then

$$
\nu_{p}(\Gamma) \leqq \frac{36.1 g H_{1} H_{2}}{E^{3}(\log p)^{4}}\left(\max \left\{\log b^{\prime}+\log (E \log p)+0.4,6 E \log p\right\}\right)^{2}
$$

and

$$
\nu_{p}(\Gamma) \leqq \frac{53.8 g H_{1} H_{2}}{E^{3}(\log p)^{4}}\left(\max \left\{\log b^{\prime}+\log (E \log p)+0.4,4 E \log p\right\}\right)^{2} .
$$

This leads to the following proposition which is easy to use.

Proposition 5.5. Let $g^{\prime}$ be a positive integer such that $\nu_{p}\left(\alpha_{1}^{g^{\prime}}-1\right)>0$ and $\nu_{p}\left(\alpha_{2}^{g^{\prime}}-1\right)>0$. Assume that there exists an integer $F$ such that

$$
2 \nu_{p}(d)<F \leqq \nu_{p}\left(\alpha_{1}^{g^{\prime}}-1\right)
$$


with $d=g^{\prime} / g$. Let $H_{1}, H_{2}$ be real numbers such that

$$
H_{i} \geqq \max \left\{\log \mathrm{h}\left(\alpha_{i}\right), F \log p\right\} \quad(i=1,2) .
$$

Put $b^{\prime}$ as in Proposition 5.4. Moreover, assume that

$$
\exp (0.4)(\log p)\left(F-\nu_{p}(d)\right) b^{\prime}<p^{4\left(F-\nu_{p}(d)\right)} .
$$

If $p \geqq 5$, then

$$
\nu_{p}(\Gamma) \leqq \frac{860.8 g^{\prime} H_{1} H_{2}}{F(\log p)^{2}} .
$$

Proof. Note that $g^{\prime}$ is a multiple of $g$. Then, by a well-known fact on $p$-adic calculations,

$$
\nu_{p}\left(\alpha_{1}^{g^{\prime}}-1\right)=\nu_{p}\left(\alpha_{1}^{g}-1\right)+\nu_{p}\left(g^{\prime} / g\right) .
$$

Thus, if $p$ is odd, then

$$
\nu_{p}\left(\alpha_{1}^{g}-1\right)=\nu_{p}\left(\alpha_{1}^{g^{\prime}}-1\right)-\nu_{p}\left(g^{\prime} / g\right) \geqq F-\nu_{p}(d) \geqq 1>\frac{1}{p-1} .
$$

One can use the second inequality stated in Proposition 5.4 with $E=F-\nu_{p}(d)$ to find that

$$
\nu_{p}(\Gamma) \leqq \frac{860.8 g^{\prime} H_{1} H_{2}}{d\left(F-\nu_{p}(d)\right)(\log p)^{2}} .
$$

Finally, observe that $d\left(F-\nu_{p}(d)\right)>d(F / 2) \geqq F$ if $d>1$.

\section{Estimates of $a$ and $b$ via linear form in logarithms}

Put

$$
\gamma=\alpha / \bar{\alpha}
$$

where $\alpha=m+i$. Also, let us consider the principal determinations of the logarithms of $\gamma$ and -1 , that is,

$$
\log \gamma=2 \theta i, \quad \log (-1)=\pi i
$$

Recall that $\theta=\operatorname{Arctan}(1 / m)$.

Lemma 6.1. Assume that

$$
m>10^{11}
$$

Put

$$
\Lambda=b_{2} \log \gamma-b_{1} \log (-1),
$$

where $b_{1}, b_{2}$ are positive integers with $b_{2} \geqq m b_{1}$. Then

$$
\log |\Lambda|>-\mathcal{W}(\log c)\left(\log b_{2}\right)^{2}
$$

with $\mathcal{W}=17.7$ 
Proof. Without loss of generality, one may assume that $b_{1}, b_{2}$ are coprime. Let us apply Proposition 5.1 with $\left(\alpha_{1}, \alpha_{2}\right)=(-1, \gamma)$. Observe that $D=1$, and

$$
\begin{aligned}
& \left|\log \alpha_{1}\right|=\pi, \quad \log \left|\alpha_{1}\right|=0, \quad \mathrm{~h}\left(\alpha_{1}\right)=0, \\
& \left|\log \alpha_{2}\right|=2 \theta, \quad \log \left|\alpha_{2}\right|=0, \quad \mathrm{~h}\left(\alpha_{2}\right)=(\log c) / 2 .
\end{aligned}
$$

Thus, let us take

$$
a_{1}=\varrho \pi, \quad a_{2}=2 \varrho \theta+\log c
$$

with $\varrho=3.86$. Set the other parameters as follows:

$$
\begin{aligned}
& \mu=1, \quad K=\left\lceil a_{1} a_{2} L\right\rceil, \quad L=\left\lceil\log b_{2}\right\rceil \\
& R_{1}=2, \quad R_{2}=\left\lceil\frac{1+(K-1) L}{S_{2}}\right\rceil, \quad S_{1}=\lceil L / 2\rceil, \quad S_{2}=\left\lceil a_{1} L\right\rceil .
\end{aligned}
$$

Let us observe both (I) and (II) of Proposition 5.1. The first inequality in (I) holds as $R_{1} S_{1} \geqq L$ and $\alpha$ is not a root of unity. In order to show the second one, suppose that

$$
u b_{2}+v b_{1}=u^{\prime} b_{2}+v^{\prime} b_{1}
$$

for some integers $u, u^{\prime}, v, v^{\prime}$ such that $0 \leqq u, u^{\prime}<R_{2}$ and $0 \leqq v, v^{\prime}<S_{2}$. As $\operatorname{gcd}\left(b_{1}, b_{2}\right)=1$, one reduces the above equation modulo $b_{2}$ to find

$$
v \equiv v^{\prime} \bmod b_{2} .
$$

Observe that

$$
(0 \leqq) \quad v, v^{\prime}<S_{2}=\left\lceil\varrho \pi\left\lceil\log b_{2}\right\rceil\right\rceil \leqq b_{2},
$$

where the last inequality holds as $b_{2}$ is not so small by the assumption that $b_{2} \geqq m b_{1}$ with $m>10^{11}$. It follows that the above congruence modulo $b_{2}$ is actually an equality, that is, $v=v^{\prime}$, hence $u=u^{\prime}$. Thus, the second inequality in (I) holds from the fact that $R_{2} S_{2} \geqq 1+(K-1) L$.

Next, let us observe the inequality in (II). As $\sigma=1$, its left-hand side is

$$
(\log \varrho) N-(\log \varrho) K-2 \log N-(K-1) \log \mathcal{B}-g L\left(R a_{1}+S a_{2}\right) .
$$

Let us estimate the fourth and fifth terms of (3) in turn. Observe that

$$
\begin{aligned}
& R=R_{1}+R_{2}-1<\frac{1+a_{1} a_{2} L^{2}}{a_{1} L}+2=a_{2} L+2+1 /\left(a_{1} L\right), \\
& S=S_{1}+S_{2}-1<a_{1} L+L / 2+1 .
\end{aligned}
$$

Also, by the inequality in [7, page 307 , line 11$]$, one easily deduces

$$
\left(\prod_{k=1}^{K-1} k !\right)^{-2 /\left(K^{2}-K\right)}<\frac{1.8}{K}
$$

for any $K \geqq 1$. These together with the assumption that $m>10^{11}$ and $b_{2} \geqq m b_{1}$, imply

$$
\mathcal{B}<\frac{(R-1) b_{2}+(S-1) b_{1}}{2} \cdot \frac{1.8}{K} \leqq \frac{0.9 b_{2}}{a_{1}} \cdot \frac{R-1+(S-1) / m}{a_{2} L}
$$




$$
\begin{aligned}
\frac{R-1+(S-1) / m}{a_{2} L} & <\frac{a_{2} L+1+1 /\left(a_{1} L\right)+a_{1} L / m+L /(2 m)}{a_{2} L} \\
& =1+a_{1} /\left(a_{2} m\right)+1 /\left(2 a_{2} m\right)+1 /\left(a_{2} L\right)+1 /\left(a_{1} a_{2} L^{2}\right) \\
& <1.001 .
\end{aligned}
$$

Moreover,

$$
\begin{gathered}
g=\frac{1}{4}-\frac{K L}{12 R S} \leqq \frac{1}{4}-\frac{1}{12} \cdot \frac{a_{2} L}{R} \cdot \frac{a_{1} L}{S} \\
\frac{a_{2} L}{R} \cdot \frac{a_{1} L}{S}>\frac{1}{1+2 /\left(a_{2} L\right)+1 /\left(a_{1} a_{2} L^{2}\right)} \cdot \frac{1}{1+1 /\left(2 a_{1}\right)+1 /\left(a_{1} L\right)} \\
> \\
0.955 .
\end{gathered}
$$

It follows that

$$
\begin{aligned}
g L\left(R a_{1}+S a_{2}\right) & =g\left(\frac{R}{a_{2} L}+\frac{S}{a_{1} L}\right) \cdot a_{1} a_{2} L^{2} \\
& <g\left(2+1 /\left(2 a_{1}\right)+1 /\left(a_{1} L\right)+2 /\left(a_{2} L\right)+1 /\left(a_{1} a_{2} L^{2}\right)\right) \cdot N \\
& <\iota N
\end{aligned}
$$

with $\iota=0.35$. To sum up, the number in (3) is greater than

$$
\begin{aligned}
& (\log \varrho) N-(\log \varrho) K-2 \log N-(K-1)\left(L-\log a_{1}\right)-\iota N \\
= & (\log \varrho-1-\iota) N-2 \log N+L+K \log \left(a_{1} / \varrho\right)-\log a_{1} \\
\geqq & (\log \varrho-1-\iota) N-2 \log N+L+a_{1} a_{2} L \log \pi-\log (\varrho \pi) \\
> & \varepsilon(N) .
\end{aligned}
$$

Therefore, the inequality in (II) holds.

Now, Proposition 5.1 gives

$$
\begin{aligned}
-(\log \varrho) N & <\log |\Lambda|+\log \max \left\{\frac{L S \mathrm{e}^{L S|\Lambda| /\left(2 b_{2}\right)}}{2 b_{2}}, \frac{L R \mathrm{e}^{L R|\Lambda| /\left(2 b_{1}\right)}}{2 b_{1}}\right\} \\
& \leqq \log |\Lambda|+\log (L T / 2)+L T|\Lambda| / 2
\end{aligned}
$$

with $T=\max \{R, S\}$. Since $T \leqq 1.1 a_{2} L$,

$$
N=K L \leqq\left(a_{1} a_{2} L+1\right) L=a_{1} a_{2} L^{2}+L,
$$

and since one may assume $|\Lambda|<(L T)^{-1}$, it follows that

$$
\begin{aligned}
\log |\Lambda| & >-(\log \varrho) N-\log (L T / 2)-L T|\Lambda| / 2 \\
& >-(\log \varrho)\left(a_{1} a_{2} L+1\right) L-\log \left(0.6 a_{2} L^{2}\right)-1 / 2 \\
& >-(\log \varrho)\left(a_{1}+\frac{L \log \rho+\log \left(0.6 a_{2} L^{2}\right)+1 / 2}{(\log \varrho) a_{2} L^{2}}\right) \cdot a_{2} L^{2} .
\end{aligned}
$$

This yields the stated inequality. 
Lemma 6.2. Assume that

$$
10^{10}<r<10^{74}
$$

Then

$$
\max \left\{W_{a}, W_{b}\right\}<\mathcal{W}(\log r)^{2}(\log c)
$$

In particular,

$$
X>\min \{x, y, 2\} \cdot \frac{r \log c-\log 2}{4 \mathcal{W}(\log r)^{2}(\log c)} .
$$

Proof. According to [4, page 256, line 9],

$$
\max \left\{W_{a}, W_{b}\right\}<\log \pi-\log |2 r \theta-j \pi|
$$

where $j$ is a non-negative integer for which $|2 r \theta-j \pi|$ is minimal. If $j=0$, then $|2 r \theta-j \pi|=$ $2 r \theta>1.8 \mathrm{r} / \mathrm{m}$. Thus, one may assume that $j>0$. In order to show the lemma, it suffices to consider when $|2 r \theta-j \pi|$ is relatively small, for example,

$$
|2 r \theta-j \pi|<\frac{\pi}{r^{10}}
$$

Since $\theta<1 / m$, it is easy to see from (4) that

$$
0<j \leqq r / m
$$

Put

$$
\Lambda=r \log \gamma-j \log (-1) .
$$

Then $\Lambda=(2 r \theta-j \pi) \sqrt{-1}$, thereby

$$
\max \left\{W_{a}, W_{b}\right\}<\log \pi-\log |\Lambda| .
$$

If $m>10^{11}$, then the stated inequality follows from Lemma 6.1 for $\left(b_{1}, b_{2}\right)=(j, r)$. Let us finish the proof by observing that inequality (4) does not hold for any $m$ satisfying $m \leqq 10^{11}$. Dividing (4) by $r \pi$ gives

$$
\left|\frac{2 \theta}{\pi}-\frac{j}{r}\right|<\frac{1}{r^{11}}<\frac{1}{2 r^{2}}
$$

By Legendre's theorem on continued fraction, $j / r$ is a convergent to $2 \theta / \pi$. Let $j / r$ be the $t$-th convergent to $2 \theta / \pi$, say $j / r=p_{t} / q_{t}$. Then

$$
\frac{1}{\left(a_{t+1}+2\right) q_{t}^{2}}<\left|\frac{2 \theta}{\pi}-\frac{j}{r}\right|<\frac{1}{r^{11}},
$$

where $a_{t+1}$ is the $(t+1)$-st partial quotient of $2 \theta / \pi$. Since $q_{t} \leqq r$, and $10^{10}<r<10^{74}$ by assumption, one has

$$
q_{t}<10^{74}, \quad a_{t+1}>r^{9}-2>10^{90} .
$$

For each even $m$ with $2 \leqq m \leqq 10^{11}$, one can use a computer (by Pari/Gp, in a week) to check that $a_{t+1}<10^{90}$ for any index $t$ satisfying $q_{t}<10^{74}$. This is a contradiction. 


\section{Linear form in two logarithms via solutions}

Put

$$
\Lambda_{0}=z \log c-\max \{x \log a, y \log b\} \quad(>0) .
$$

Then

$$
0<\Lambda_{0}<\log 2
$$

Also, define $\mathcal{S}$ as follows:

$$
\mathcal{S}= \begin{cases}y / \log c & \text { if } a^{x}<b^{y}, \\ x / \log c & \text { if } a^{x}>b^{y}\end{cases}
$$

Lemma 7.1. Assume that

$$
r>10^{10}, \quad \mathcal{S}>10^{7} .
$$

If $r>10^{14}$ or $m>10^{10}$, then

$$
\log \Lambda_{0}> \begin{cases}-\mathcal{C}_{1}(\log b)(\log c) h^{2} & \text { if } a^{x}<b^{y}, \\ -\mathcal{C}_{1}(\log a)(\log c) h^{2} & \text { if } a^{x}>b^{y}\end{cases}
$$

with $\mathcal{C}_{1}=13$, where

$$
h=\log (2 \mathcal{S}+1)+2.143 .
$$

Proof. Consider the case where $a^{x}<b^{y}$. Then

$$
\Lambda_{0}=z \log c-y \log b
$$

Since $c^{z}=a^{x}+b^{y}<2 b^{y}$, it follows that

$$
\frac{z}{\log b}<\frac{y}{\log c}+1=\mathcal{S}+1 .
$$

Let us apply Proposition 5.2 with $\left(\alpha_{1}, \alpha_{2}\right)=(b, c)$ and $\left(b_{1}, b_{2}\right)=(y, z)$. Set $(\varrho, \mu)=(5.79,0.63)$. Put

$$
a_{1}=(\varrho+1) \log b, \quad a_{2}=(\varrho+1) \log c .
$$

In what follows, let us consider only the case where $r>10^{14}$. Then

$$
\log b>\left(r / 2-17.7 \log ^{2} r\right) \log c>8 \cdot 10^{13}
$$

from Lemma 6.2. Define $h$ as stated. Let us check the required inequality condition on $h$. Observe that

$$
\begin{aligned}
\log \left(\frac{b_{1}}{a_{2}}+\frac{b_{2}}{a_{1}}\right) & =\log \left(\frac{y}{\log c}+\frac{z}{\log b}\right)-\log (\varrho+1) \\
& <\log (2 \mathcal{S}+1)-\log (\varrho+1) \\
& <h-\log \lambda-1.81-\log \varrho .
\end{aligned}
$$


Also, the inequality $h \geqq \max \{\lambda,(\log 2) / 2\}+\log \varrho$ holds as $\mathcal{S}$ is not extremely small. To sum up, Proposition 5.2 gives

$$
\log \Lambda_{0}>-\left(1+c_{1}+c_{2}\right) \mathcal{H}
$$

where

$$
\begin{aligned}
\mathcal{H} & =C(\varrho+1)^{2}(\log b)(\log c) h^{2}, \\
c_{1} & =\frac{\sqrt{\omega \theta}}{\mathcal{H} / h}, \quad c_{2}=\frac{\log \left(\left(C^{\prime} / C\right) \mathcal{H}\right)}{\mathcal{H}} .
\end{aligned}
$$

Let us estimate $C, c_{1}, c_{2}$ from above. For this, observe that

$$
H=h / \lambda>11.58 \text {. }
$$

Then

$$
\omega=2+2 \sqrt{1+\frac{1}{4 H^{2}}}<4.002, \quad \theta=\sqrt{1+\frac{1}{4 H^{2}}}+\frac{1}{2 H}<1.045 .
$$

These inequalities together yield that

$$
C_{0}=\left(\frac{\omega}{6}+\frac{1}{2} \sqrt{\frac{\omega^{2}}{9}+\frac{8 \lambda \omega^{5 / 4} \theta^{1 / 4}}{3 \sqrt{a_{1} a_{2} H}}+\frac{4}{3}\left(\frac{1}{a_{1}}+\frac{1}{a_{2}}\right) \frac{\lambda \omega}{H}}\right)^{2}<1.82 .
$$

Then

$$
\begin{aligned}
& C=C_{0} \mu /\left(\lambda^{3} \sigma\right)<0.281, \\
& C>\omega^{2} / 9 \cdot \mu /\left(\lambda^{3} \sigma\right)>0.274, \\
& C^{\prime}=\sqrt{C_{0} \omega \theta / \lambda^{6}}<0.63 .
\end{aligned}
$$

Thus, both $c_{1}, c_{2}$ are extremely small, more precisely,

$$
\begin{aligned}
c_{1} & <\frac{\sqrt{\omega \theta}}{\mathcal{H} / h}<\frac{2.1}{C(\varrho+1)^{2}(\log b)(\log c) h}<7 / 10^{17} \\
c_{2} & <\frac{\log (3 \mathcal{H})}{\mathcal{H}}<8 / 10^{17} .
\end{aligned}
$$

The case where $a^{x}>b^{y}$ is dealt with similarly.

\section{Bounds for solutions in terms of $r$ and $m$}

Lemma 8.1. Assume that

$$
r>10^{10} \text {. }
$$

Then the following inequalities hold.

$$
\begin{cases}y<2.001 x & \text { if } a^{x}>b^{y / 2}, \\ x<2.001 y & \text { if } a^{x / 2}<b^{y}, \\ X<5040 \log c & \text { if } a^{x}<b^{y / 2} \text { or } a^{x / 2}>b^{y} .\end{cases}
$$


Proof. Let us use Lemma 6.2. Note that

$$
\frac{r}{2}-\mathcal{W}(\log r)^{2}>0
$$

If $a^{x}>b^{y / 2}$, then $b^{y}<\left(a^{2}\right)^{x}<c^{r x}$, and so

$$
y<\frac{r x \log c}{\log b}<\frac{r x \log c}{(r / 2) \log c-\mathcal{W}(\log r)^{2} \log c}=\frac{x}{1 / 2-\mathcal{W}(\log r)^{2} / r}<2.001 x .
$$

The case where $a^{x / 2}<b^{y}$ is dealt with similarly. Next, consider the case where $a^{x}<b^{y / 2}$. Then

$$
x<\frac{y \log b}{2 \log a}<\frac{y \log c^{r / 2}}{r \log c-2 \mathcal{W}(\log r)^{2} \log c}=\frac{y}{2-4 \mathcal{W}(\log r)^{2} / r}<0.6 y .
$$

On the other hand,

$$
\Lambda_{0}=z \log c-y \log b, \quad \mathcal{S}=\frac{y}{\log c} .
$$

Since $\exp \left(\Lambda_{0}\right)=c^{z} / b^{y}=1+a^{x} / b^{y}$, it follows that

$$
(0<) \quad \Lambda_{0}=\log \left(1+a^{x} / b^{y}\right)<a^{x} / b^{y}<b^{-y / 2} .
$$

Thus,

$$
\log \Lambda_{0}<-\frac{\log b}{2} y
$$

In particular, $y=X$. On the other hand, let us apply Proposition 5.3 with $\left(\alpha_{1}, \alpha_{2}\right)=(b, c)$ and $\left(b_{1}, b_{2}\right)=(y, z)$. One may take $\left(H_{1}, H_{2}\right)=(\log b, \log c)$. Then

$$
\log \Lambda_{0}>-25.2(\log b)(\log c)\left(\max \left\{\log b^{\prime}+0.38,10\right\}\right)^{2},
$$

where

$$
b^{\prime}=\frac{y}{\log c}+\frac{z}{\log b}<2 \mathcal{S}+1 .
$$

Thus, one combines these upper and lower bounds for $\log \Lambda_{0}$ to obtain

$$
\mathcal{S}<50.4(\max \{\log (2 \mathcal{S}+1)+0.38,10\})^{2} .
$$

This implies that $\mathcal{S}<5040$, that is, $X<5040 \log c$. The case where $a^{x / 2}>b^{y}$ is dealt with similarly.

Lemma 8.2. Assume that

$$
r>10^{10}
$$

Then

$$
\min \{x, y\} \geqq 4 \text {. }
$$

Proof. Suppose that $\min \{x, y\}<4$. Then Lemma 8.1 yields that

$$
X<5040 \log c
$$


If $r>m$, then Lemma 6.2 yields

$$
r<\frac{\log 2}{\log c}+4 \mathcal{W}(\log r)^{2}(\log c) \cdot 5040<\frac{\log 2}{\log 5}+4 \mathcal{W}(\log r)^{3} \cdot 2 \cdot 5040
$$

This gives $r<9 \cdot 10^{9}$. Similarly, by Lemma 3.3 and the assumption that $m<10^{34}$, if $m \geqq r$ then

$$
r<\frac{\log 2}{\log c}+4 \log (2 m / r) \cdot 5040<\frac{\log 2}{\log r^{2}}+4 \log \left(2 \cdot 10^{34} / r\right) \cdot 5040,
$$

so $r<2 \cdot 10^{6}$. Therefore, in either case, one finds a contradiction to the assumption that $r>10^{10}$. This shows that $x \geqq 4$. It is similarly shown that $y \geqq 4$.

Lemma 8.3. Assume that

$$
10^{10}<r<10^{74}, \quad m<10^{34} .
$$

Then

$$
X<31.4 r(\log c)^{2}(\log (82 r \log c))^{2} .
$$

Proof. Consider the case where $r$ is odd. Note that $a$ is even. By Lemma 8.1, one may assume that $a^{x}>b^{y / 2}$, and

$$
X<2.0001 x \text {. }
$$

On the other hand, put

$$
\Gamma=c^{z} b^{-y}-1 .
$$

Then $\nu_{2}(\Gamma) \geqq x$. Let us apply Proposition 5.4 for $p=2$ with $\left(\alpha_{1}, \alpha_{2}\right)=\left(c,(-1)^{(b-1) / 2} / b\right)$ and $\left(b_{1}, b_{2}\right)=(z, y)$. Observe that $g=1$, and one may take $E=2$ and $\left(H_{1}, H_{2}\right)=(\log c,(r / 2) \log c)$. Then

$$
x \leqq \nu_{2}(\Gamma)<9.8 r(\log c)^{2}\left(\log \max \left\{b^{\prime}(\log 4) \exp (0.4), 2^{12}\right\}\right)^{2}
$$

where

$$
b^{\prime}=\frac{2 z}{r \log c}+\frac{y}{\log c} .
$$

Since $y<2.0001 x$ and $c^{z}=a^{x}+b^{y}<2 a^{2 x}<2 c^{r x}$, it follows that

$$
b^{\prime}<\frac{r x}{(r / 2) \log c}+\frac{2.0001 x}{\log c}<\frac{4.01 x}{\log c} .
$$

Put

$$
s=\frac{8.3 x}{\log c} \quad\left(>b^{\prime} \cdot(\log 4) \cdot \exp (0.4)\right)
$$

Then

$$
x \leqq 9.8 r(\log c)^{2}\left(\log \max \left\{s, 2^{12}\right\}\right)^{2} .
$$

If $s<2^{12}$, then

$$
X<2.0001 x=2.0001 \cdot(s / 8.3) \log c<988 \log c .
$$

If $s \geqq 2^{12}$, then

$$
\frac{s}{(\log s)^{2}} \leqq A
$$


with $A=81.34 r \log c$. Since $A$ is large $\left(>4 ? 3.5 ? \cdot 10^{12}\right)$, one has

$$
s<1.6 A(\log A)^{2}<131 r(\log c)(\log (82 r \log c))^{2} .
$$

Therefore,

$$
X<2.001 x=(2.001 / 8.3) s \log c<31.4 r(\log c)^{2}(\log (82 r \log c))^{2} .
$$

Similarly, the case where $r$ is even is dealt with by using the fact that $b$ is even and considering the cases where $b^{y}<a^{x / 2}$ and $b^{y} \geqq a^{x / 2}$ separately.

\section{Case where $m \geqq r$}

Throughout this section, assume that

$$
r>10^{10}, \quad m \geqq r, \quad m<10^{34} .
$$

Lemma 9.1. The following inequalities hold.

(i) $X>2(c-1) / r^{2}$.

(ii) $r>c^{1 / 3.81}$.

Proof. (i) Note that $m>2$. Also, $\min \{x, y\} \geqq 4$ by Lemma 8.3. Thus, the statement is true by [13, Lemma 3.3 (i)].

(ii) The first statement and Lemma 8.3 together give

$$
c-1<15.7 r^{3}(\log c)^{2}(\log (82 r \log c))^{2} .
$$

Since $r>10^{10}$, if $r \leqq c^{1 / 3.81}$ then

$$
c>10^{38.1}, \quad c-1<15.7 c^{3 / 3.81}(\log c)^{2}\left(\log \left(82 c^{1 / 3.81} \log c\right)\right)^{2} .
$$

However, these are not compatible.

Lemma 9.2. Let $p$ be any prime factor of $c$. Then

$$
z<1721.6 f(p) r|x-y|
$$

where

$$
f(p)=\frac{(\log c) \log \max \left\{c^{1 / 2}, p\right\}}{(\log p)^{2}}
$$

is a decreasing function on $p$.

Proof. Consider the case where $x>y$. Put

$$
\Gamma=a^{2(x-y)}(a / b)^{2 y}-1 .
$$


Then $\nu_{p}(\Gamma) \geqq z$. Let us apply Proposition 5.5 with $\left(\alpha_{1}, \alpha_{2}\right)=\left(a^{2}, a / b\right)$ and $\left(b_{1}, b_{2}\right)=(\mid x-$ $y \mid, 2 y)$. Note that

$$
a^{x} \equiv-b^{y} \quad \bmod c^{z}, \quad a^{2} \equiv-b^{2} \quad \bmod c^{r} .
$$

Since $x \equiv y(\bmod 2)$ by [14, Lemma 1$]$, and one may clearly assume that $z \geqq r$, it follows that

$$
\begin{aligned}
& a^{4 x} \equiv b^{4 y} \equiv\left(-a^{2}\right)^{2 y} \equiv a^{4 y} \bmod c^{r} \\
& a^{2|x-y|} \equiv\left(-b^{2}\right)^{|x-y|} \equiv b^{2|x-y|} \bmod c^{r} .
\end{aligned}
$$

Thus,

$$
\alpha_{i}^{2|x-y|} \equiv 1 \quad \bmod p^{r} \quad(i=1,2)
$$

Put $g^{\prime}=2|x-y|$. Let us take

$$
F=r, \quad H_{1}=r \log c, \quad H_{2}=\max \{(r / 2) \log c, r \log p\} .
$$

Since $d=g^{\prime} / g \leqq g^{\prime} / 2=|x-y|<X$, it follows from Lemma 8.3 that

$$
\nu_{p}(d)<\frac{\log X}{\log p}<r / 2
$$

Also, since

$$
b^{\prime}=\frac{|x-y|}{H_{2}}+\frac{2 y}{r \log c}<\frac{X}{(r / 2) \log c}+\frac{2 X}{r \log c}=\frac{4 X}{r \log c},
$$

one finds that

$$
\exp (0.4)(\log p)\left(r-\nu_{p}(d)\right) b^{\prime}<4 \exp (0.4) X<5^{2 r}<p^{4\left(r-\nu_{p}(d)\right)}
$$

Therefore, Proposition 5.5 gives the stated inequality. Similarly, the case where $x<y$ is dealt with by examining the $p$-adic value of $b^{2(y-x)}(b / a)^{2 x}-1$.

\section{Lemma 9.3.}

$$
|x-y|<\frac{2\left|\log \Lambda_{0}\right|}{r \log c}+\frac{2 \max \left\{W_{a}, W_{b}\right\}}{r \log c} X .
$$

Proof. Consider the case where $a^{x}<b^{y}$. Since $\exp \left(\Lambda_{0}\right)=c^{z} / b^{y}=1+a^{x} / b^{y}$, it follows that

$$
(0<) \quad \Lambda_{0}=\log \left(1+a^{x} / b^{y}\right)<a^{x} / b^{y}<1 .
$$

Thus,

$$
\left|\log \Lambda_{0}\right|=-\log \Lambda_{0}>y \log b-x \log a=\frac{r \log c}{2}(y-x)+R>0,
$$

where $R=W_{a} x-W_{b} y$. Observe that

$$
\frac{r \log c}{2}|x-y|<\left|\log \Lambda_{0}\right|+|R|, \quad|R|<\max \left\{W_{a}, W_{b}\right\} X .
$$

Therefore, these inequalities together give the stated inequality. The case where $a^{x}>b^{y}$ is dealt with similarly. 
Lemma 9.4. Assume that $\mathcal{S}>10^{7}$. Then the following hold.

(i) Any prime factor of $c$ is greater than $r^{2.79 / 5}$.

(ii) The total number of prime factors of $c$ is at most 6 .

Proof. Let $p$ be any prime factor of $c$. If $p>r^{2.79 / 5}$, then Lemma 9.1 (ii) leads to

$$
p>c^{(1 / 3.81) \cdot(2.79 / 5)}>c^{1 / 7} .
$$

Thus, it suffices to show only the first statement. It is shown in the proof of [13, Lemma 3.5] that

$$
r \leqq \frac{192 p \log c}{(\log p)^{3}}\left(\max \left\{\log b^{\prime}+\log \log p+0.4, \log p^{5}\right\}\right)^{2},
$$

where

$$
b^{\prime}=\frac{8(r+1)|x-y|}{\log p} .
$$

In view of Lemmas 4.2, 7.1 and 9.3, one observes that

$$
\begin{aligned}
|x-y| & <\frac{\left|\log \Lambda_{0}\right|}{\log c^{r / 2}}+\frac{2 \log (2 m / r)}{r \log c} X \\
& <\mathcal{C}_{1}(\log c)(\log (2 \mathcal{S}+1)+2.143)^{2}+X / r .
\end{aligned}
$$

This together with Lemma 8.3 gives

$$
b^{\prime} \cdot(\log p) \cdot \exp (0.4)<8 \exp (0.4)(r+1)|x-y|<r^{e},
$$

where $e$ is any number satisfying $e \geqq 2$. It follows from (5) that

$$
r \leqq \frac{192 p \log c}{(\log p)^{3}}\left(\log \max \left\{r^{e}, p^{5}\right\}\right)^{2}
$$

If $p^{5} \leqq r^{e}$, then

$$
\frac{r}{(\log r)^{2}} \leqq \frac{192 e^{2} p \log c}{(\log p)^{3}} \leqq \frac{192 e^{2} r^{e / 5} \log c}{\left(\log r^{e / 5}\right)^{3}}=\frac{5^{3} \cdot 192 r^{e / 5} \log c}{e(\log r)^{3}}
$$

so Lemma 9.1 (i) yields

$$
e r^{1-e / 5} \log r<\left(5^{3} \cdot 192\right) \log \left(r^{2} X / 2+1\right)
$$

Lemma 8.3 enables us to take $e=2.79$ to find that this inequality does not hold. Thus, $p^{5}>r^{2.79}$.

Lemma 9.5. $X<4.2 \cdot 10^{8} \log c$.

Proof. Firstly, from Lemma 6.2 observe that

$$
X<\frac{\log \max \{a, b\}}{\log \min \{a, b\}} \cdot \mathcal{S} \log c<\frac{1}{1-2 \mathcal{W}(\log r)^{2} / r} \cdot \mathcal{S} \log c<2 \mathcal{S} \log c .
$$


Thus one may assume that $\mathcal{S}>10^{7}$. Then the largest prime factor of $c$ is at least $c^{1 / 6}$ by Lemma 9.4 (ii). Lemma 9.2 for that prime gives

$$
z<\mathcal{C}_{2} r|x-y|
$$

with $\mathcal{C}_{2}=860.8 \cdot 6^{2}=30988.8$. Then, by inequality (6),

$$
z<\mathcal{C}_{1} \mathcal{C}_{2} r(\log c)(\log (2 \mathcal{S}+1)+2.143)^{2}+\mathcal{C}_{2} X
$$

On the other hand, by Lemma 4.2,

$$
z>\frac{\log \min \{a, b\}}{\log c} X>\left(r / 2-\frac{\log (2 m / r)}{\log c}\right) X .
$$

These upper and lower bounds for $z$ together imply

$$
\left(r / 2-\frac{\log (2 m / r)}{\log c}-\mathcal{C}_{2}\right) \mathcal{S}^{\prime}<\mathcal{C}_{1} \mathcal{C}_{2} r\left(\log \left(2 \mathcal{S}^{\prime}+1\right)+2.143\right)^{2}
$$

with $\mathcal{S}^{\prime}=X / \log c$. Since the left-hand side above is positive, it follows that

$$
\frac{\mathcal{S}^{\prime}}{\left(\log \left(2 \mathcal{S}^{\prime}+1\right)+2.143\right)^{2}}<\frac{\mathcal{C}_{1} \mathcal{C}_{2}}{1 / 2-\frac{\log (2 m / r)}{r \log c}-\mathcal{C}_{2} / r}<2.0001 \mathcal{C}_{1} \mathcal{C}_{2}
$$

This implies that $\mathcal{S}^{\prime}<4.2 \cdot 10^{8}$.

Lemmas 9.1 (i) and 9.5 together imply that

$$
c-1<2.1 \cdot 10^{8} r^{2} \log c .
$$

A similar observation in the proof of Lemma 9.1 (ii) yields that $r>c^{1 / 3.11}$. Thus, by Lemma 9.4 (i), any prime factor of $c$ is greater than $c^{(1 / 3.11) \cdot(2.79 / 5)}>c^{1 / 6}$, in particular, the largest prime factor of $c$ is at least $c^{1 / 5}$. A similar observation in the proof of Lemma 9.5 yields that

$$
X<2.8 \cdot 10^{8} \log c .
$$

This together with Lemma 9.1 (i) implies

$$
2 \log (m / r)<\log \left(1.4 \cdot 10^{8}\right)+\log \log c
$$

In view of Lemmas 3.3, 4.2 and 8.2,

$$
X>\frac{r \log c-\log 2}{2 \log (2 m / r)}>\frac{r \log c-\log 2}{\log 4+\log \left(1.4 \cdot 10^{8}\right)+\log \log c} .
$$

Comparing the obtained upper and lower bounds for $X$ gives

$$
r<\frac{\log 2}{\log c}+\left(\log 4+\log \left(1.4 \cdot 10^{8}\right)+\log \log c\right) \cdot 2.8 \cdot 10^{8} .
$$

This implies that $r<7.4 \cdot 10^{9}$, which contradicts the assumption that $r>10^{10}$. This contradiction completes the proof of Theorem 1.2 (I). 


\section{Case where $r>m$}

Proof of Theorem 1.2(II). The proof goes along similar lines to that of (I). Assume that

$$
r>10^{14}, \quad r>m, \quad m<10^{34} .
$$

Firstly, let us observe that the total number of prime factors of $c$ is at most 2 . Note that $r \geqq m+1>c^{1 / 2}$. Let $p$ be any prime factor of $c$. If $p>r^{3.52 / 5}$, then $p>r^{2 / 3}>c^{1 / 3}$. Thus, it suffices to show that $p \geqq r^{3.52 / 5}$. For this, observe from Lemmas 6.2, 7.1 and 9.3 that

$$
|x-y|<\mathcal{C}_{1}(\log c)(\log (2 \mathcal{S}+1)+2.143)^{2}+2 \mathcal{W} r^{-1}(\log r)^{2} X .
$$

On inequality (5), the above inequality together with Lemma 8.3 gives

$$
b^{\prime} \cdot(\log p) \cdot \exp (0.4)<r^{e},
$$

where $e$ is any number satisfying $e \geqq 3$. As seen in the proof of Lemma 9.4, if $p^{5} \leqq r^{e}$, then

$$
e r^{1-e / 5}<5^{3} \cdot 192(\log c) / \log r<5^{3} \cdot 192 \cdot 2 .
$$

Since $r>10^{14}$, one may take $e=3.52$ to find that the above inequalities do not hold. Thus, $p^{5}>r^{3.52}$.

Since the largest prime factor of $c$ is at least $c^{1 / 2}$, one uses Lemma 9.2 for that prime to find that

$$
z<\mathcal{C}_{2} r|x-y|
$$

with $\mathcal{C}_{2}=860.8 \cdot 4=3443.2$. Then, by (7),

$$
z<\mathcal{C}_{1} \mathcal{C}_{2} r(\log c)(\log (2 \mathcal{S}+1)+2.143)^{2}+2 \mathcal{C}_{2} \mathcal{W}(\log r)^{2} X .
$$

On the other hand, one knows from Lemma 6.2 that

$$
z>\frac{\log \min \{a, b\}}{\log c} X>\left(r / 2-\mathcal{W}(\log r)^{2}\right) X .
$$

These inequalities for $z$ together imply

$$
\left(r / 2-\left(2 \mathcal{C}_{2}+1\right) \mathcal{W}(\log r)^{2}\right) \mathcal{S}^{\prime}<\mathcal{C}_{1} \mathcal{C}_{2} r\left(\log \left(2 \mathcal{S}^{\prime}+1\right)+2.143\right)^{2} .
$$

Since the left-hand side above is positive, it follows that

$$
\frac{\mathcal{S}^{\prime}}{\left(\log \left(2 \mathcal{S}^{\prime}+1\right)+2.143\right)^{2}}<\frac{\mathcal{C}_{1} \mathcal{C}_{2}}{1 / 2-\left(2 \mathcal{C}_{2}+1\right) \mathcal{W}(\log r)^{2} / r}<2.0001 \mathcal{C}_{1} \mathcal{C}_{2}
$$

This implies that $\mathcal{S}^{\prime}<3.7 \cdot 10^{7}$. Therefore, Lemma 3.3 gives

$$
\frac{r \log c-\log 2}{2 \mathcal{W}(\log r)^{2} \log c}<X<3.7 \cdot 10^{7} \log c
$$

thereby

$$
r<(\log 2) / \log c+2 \mathcal{W}(\log r)^{2}(\log c) \cdot 3.7 \cdot 10^{7} .
$$

Since $c<r^{2}$, the above inequality gives $r<8.9 \cdot 10^{13}$, which contradicts the assumption that $r>10^{14}$. This contradiction completes the proof of Theorem 1.2(II). 


\section{Proof of Theorem 1.1}

By Theorem 1.2, it suffices to prove Conjecture 1.2 in the case where

$$
r \leqq 10^{10}, \quad m>10^{23}
$$

Suppose that there exists a solution $(x, y, z)$ of equation (2) with $\Delta>0$. Since the inequality $\mathcal{W}(\log r)^{2} \log c>\log (2 m / r)$ clearly holds, and it may be assumed that $r \geqq(m / 2)^{1 / 3}>3.6 \cdot 10^{7}$ by [9, Theorem 1], one can show the same conclusions as those of Lemmas 8.1, 8.2, 8.3 and 9.1 (i). Then one uses the bounds for $X$ obtained in Lemmas 8.3 and 9.1 (i) to conclude that

$$
c-1<15.7 r^{3}(\log c)^{2}(\log (82 r \log c))^{2} .
$$

Since $r \leqq 10^{10}$, this implies that $c<2 \cdot 10^{38}$, so $m<10^{20}$. This contradiction completes the proof of Theorem 1.1.

\section{Acknowledgements}

The author would like to thank Professor Mihai Cipu for his comments. This work is supported by Grant-in-Aid for Young Scientists (B) (No.16K17557).

\section{References}

[1] Bugeaud, Y. (1999). Linear forms in $p$-adic logarithms and the Diophantine equation $\left(x^{n}-1\right) /(x-1)=y^{q}$. Math. Proc. Cambridge Phil. Soc., 127 (3), 373-381.

[2] Cao, Z. \& Dong, X. (2002). On the Terai-Jeśmanowicz conjecture. Publ. Math. Debrecen, 61 (3-4), 253-265.

[3] Cao, Z. \& Dong, X. (2003). An application of a lower bound for linear forms in two logarithms to the Terai-Jeśmanowicz conjecture. Acta Arith., 110 (2), 153-164.

[4] Cipu, M. \& Mignotte, M. (2009). On a conjecture on exponential Diophantine equations. Acta Arith., 140 (3), 251-269.

[5] Jeśmanowicz, L. (1955/56). Several remarks on Pythagorean numbers. Wiadom. Mat., 1 (2), 196-202 (in Polish).

[6] Laurent, M. (2008). Linear forms in two logarithms and interpolation determinants II. Acta Arith., 133 (4), 325-348.

[7] Laurent, M., Mignotte, M. \& Nesterenko, Y. (1995). Formes linéaires en deux logarithmes et déterminants dínterpolation. J. Number Theory, 55 (2), 285-321.

[8] Le, M. (2003). A conjecture concerning the exponential Diophantine equation $a^{x}+b^{y}=c^{z}$. Acta Arith., 106 (4), 345-353. 
[9] Le, M., Togbe, A. \& Zhu, H. (2014). On a pure ternary exponential Diophantine equation. Publ. Math. Debrecen, 85 (3-4), 395-411.

[10] Lu, W. (1959). On the Pythagorean numbers $4 n^{2}-1,4 n$ and $4 n^{2}+1$. Acta Sci. Natur. Univ. Szechuan, 2, 39-42 (in Chinese).

[11] Luca, F. (2012). On the system of Diophantine equations $a^{2}+b^{2}=\left(m^{2}+1\right)^{r}$ and $a^{x}+b^{y}=$ $\left(m^{2}+1\right)^{z}$. Acta Arith., 153 (4), 373-392.

[12] Miyazaki, T. (2011). Terai's conjecture on exponential Diophantine equations. Int. J. Number Theory, 7 (4), 981-999.

[13] Miyazaki, T. (2014). A note on the article by F. Luca "On the system of Diophantine

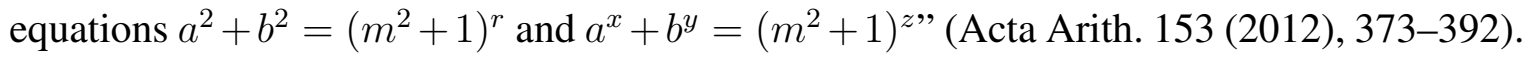
Acta Arith., 164 (1), 31-42.

[14] Scott, R. \& Styer, R. (2016). Number of solutions to $a^{x}+b^{y}=c^{z}$. Publ. Math. Debrecen, $88(1-2), 131-138$.

[15] Terai, N. (1994). The Diophantine equation $a^{x}+b^{y}=c^{z}$. Proc. Japan Acad. Ser. A Math. Sci., 70 (1), 22-26.

[16] Terai, N. (1995). The Diophantine equation $a^{x}+b^{y}=c^{z}$ II. Proc. Japan Acad. Ser. A Math. Sci., 71 (6), 109-110.

[17] Terai, N. (1996). The Diophantine equation $a^{x}+b^{y}=c^{z}$ III. Proc. Japan Acad. Ser. A Math. Sci., 72 (1), 20-22.

[18] Terai, N. (1999). Applications of a lower bound for linear forms in two logarithms to exponential Diophantine equations. Acta Arith., 90 (1), 17-35. 